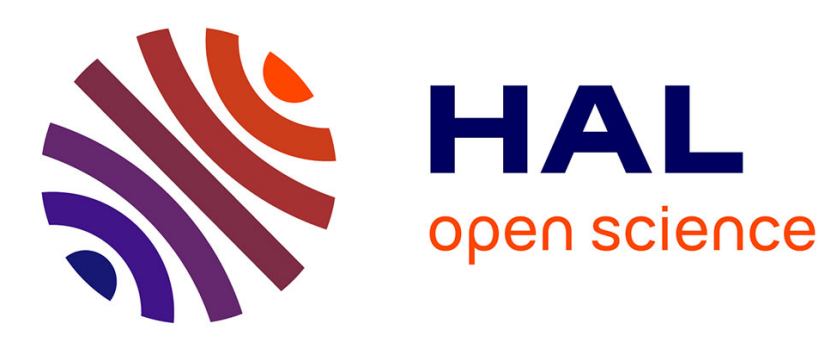

\title{
Le concept de développement: la fin d'un mythe
}

Alain Francois

\section{To cite this version:}

Alain Francois. Le concept de développement: la fin d'un mythe. L'Information géographique, 2003, 66, pp.323-336. 10.3406/ingeo.2003.2910 . hal-01364698

\section{HAL Id: hal-01364698 \\ https://hal.science/hal-01364698}

Submitted on 12 Sep 2016

HAL is a multi-disciplinary open access archive for the deposit and dissemination of scientific research documents, whether they are published or not. The documents may come from teaching and research institutions in France or abroad, or from public or private research centers.
L'archive ouverte pluridisciplinaire HAL, est destinée au dépôt et à la diffusion de documents scientifiques de niveau recherche, publiés ou non, émanant des établissements d'enseignement et de recherche français ou étrangers, des laboratoires publics ou privés. 


\title{
Le concept de développement : la fin d'un mythe
}

\author{
Alain François, Maître de conférences en géographie \\ IUFM de Poitou-Charentes, laboratoire ICoTEM (Université de Poitiers)
}

L’Information géographique, nº6, pp. 323-336, 2003.

Pays sous-développés, en voie de développement, en développement, les moins avancés... force est de constater que le concept de développement relève de subtilités de langage toujours délicates à manier. A force d'expressions hasardeuses, on en viendrait même à produire un fâcheux contre-sens en opposant aujourd'hui aux pays en développement des pays développés...qui ne se développeraient plus.

Mais l'objet de cet article n'est pas de savoir comment parler des problèmes de développement devant la diversité grandissante des situations qui se font jour au «Sud». Le sujet a déjà été abordé à maintes reprises ${ }^{1}$. Ce n'est pas ici le comment mais le pourquoi qui nous préoccupe. Voilà en effet une lecture du monde et de ses humanités qui ne va pas de soi, mais devenue si ordinaire et à ce point intégrée à nos représentations que l'on pourrait juger malvenu, voire suspect, de vouloir aujourd'hui en contester la pertinence. Pourtant, la question est ouvertement posée depuis les années 1980 par divers courants de la géographie anglo-

\footnotetext{
1 Sylvie Brunel, Le Sud dans la nouvelle économie mondiale, PUF, Paris, 1995, 406 p., voir également Robert Chapuis, Les quatre mondes du Tiers Monde, Masson géographie, Paris, 1994, 234 p.
}

saxonne ${ }^{2}$. En France, la polémique fut lancée en 1986 par Serge Latouche avec Faut-il refuser le développement ? 3 , puis reprise en 1996 avec la parution de l'ouvrage de Gilbert Rist Le développement. Histoire d'une croyance occidentale 4 .

Si le sujet suscite depuis les années 1990 quelques réactions et premières prises de position, au demeurant très partagées, de la part des géographes français, il présente au moins le mérite de rappeler que le concept de développement promeut une mise en ordre du monde qui ne fait plus désormais l'unanimité en science sociale. A vrai dire, celle-ci est discutée depuis la fin des années 1960 par les sociologues, les anthropologues, les ethnologues, les économistes, les juristes ainsi que par certains géographes à l'instar d'Eric Dardel, de Pierre Gourou et de Joël Bonnemaison. Plus récemment encore, Augustin Berque et Denis Retaillé relevaient l'illusion de

\footnotetext{
2 Pour une synthèse critique des courants actuels de la géographie anglo-saxonne voir K.J. Anderson \& ali, Géographies anglo-saxonnes. Tendances contemporaines, Belin, Paris, Mappemonde, 2001, $313 \mathrm{p}$.

${ }^{3}$ Serge Latouche, Faut-il refuser le développement?, PUF, Paris, 1986.

4 Gilbert Rist, Le développement. Histoire d'une croyance occidentale, Presses de Sciences Po, 2001 ( $2^{\text {ème }}$ édition), $443 \mathrm{p}$.
} 
l'universalité des valeurs qui fondent le positivisme scientifique dans la pensée moderne, reprenant par là l'un des arguments majeurs des critiques adressées aujourd'hui au « développementalisme» 5 .

\section{CONSTRUCTION ET DÉCONSTRUCTION D'UN CONCEPT}

Notre intention n'est pas ici de verser dans un scepticisme à la mode sur les motivations désintéressées de la connaissance et des sciences, mais elle n'est pas non plus d'œuvrer pour la naturalisation du savoir en science sociale. Il s'agit plutôt de rappeler que le géographe et son propos s'inscrivent irrémédiablement dans le champ

5. Augustin Berque, Etre humain sur la terre, Gallimard, Paris, 1996, (pp.27-28) : «Le premier [anthropo-centrisme] est celui de toutes les civilisations humaines avant la révolution scientifique $\mathrm{du}$ XVIIème siècle, à savoir la vision du monde ethnocentrique particulière à chaque culture. [...] Le deuxième anthropocentrisme est une particularité de la culture occidentale dont l'origine remonte à l'Antiquité grecque, qui s'est renforcée avec le christianisme, puis avec l'humanisme de la Renaissance, et qui a fini par engendrer le sujet individuel moderne : cet être qui s'institue lui-même indépendamment du monde, qui met le monde entier en perspective sous son regard, et qui en ce sens est bien le centre du monde [...] [Cet] anthropocentrisme [...] est un idéal abstrait [...] propre à la modernité ; c'est le complément nécessaire du cosmocentrisme de la science moderne $\gg$.

- Denis Retaillé, Le monde du géographe, Presses de Sciences Po, Paris, (p.31), 1997: «Avec le progrès de la connaissance par l'accès à la diversité du monde, le rapport de la croyance et du savoir s'est brouillé. Une illusion s'est installé qui laisse croire que l'universalité européenne est affranchie de la culture. C'est que l'abstraction des représentations du monde et de la terre dont les Européens se sont dotés a été d'une redoutable efficacité. Elle a simplement permis la conquête de la terre en faisant de chaque lieu un lieu égal à tout autre $[. .]$.$» .$ de l'histoire mouvementée des hommes dans leurs rapports à l'Autre.

\section{L'invention du développement}

Le « développement», entendu comme phénomène géographique global 6 , est un concept relativement récent. Il est encore totalement absent des manuels scolaires de l'immédiate après-guerre consacrés à l'Afrique, à l'Asie et à l'Amérique latine. $\mathrm{Au}$ mieux parle t-on en 1963 du développement de l'instruction et de «projets d'équipement hydroélectrique qui doivent permettre le développement économique de la Guinée ${ }^{7}$. Aucune allusion n'est encore faite aux niveaux de développement, aux problèmes de développement des jeunes Etats indépendants ou en passe de le devenir. Même si la faiblesse des équipements, l'absence de capitaux, les difficultés de mise en valeur agricole et l'accroissement démographique sont évoqués, ils le sont toujours séparément et pour eux-mêmes. Les synthèses et résumés qui apparaissent en fin de chapitres sur les populations et les sociétés adoptent un discours plus volontiers centré sur le dualisme progrès/tradition, civilisés/primitifs pour justifier plus aisément, sans doute, l'entreprise coloniale.

C'est à l'occasion du discours d'investiture du Président Truman prononcé le 20 janvier 1949 que l'expression «sousdéveloppé " est employée officiellement pour la première fois 8 . Le contexte de reconstruction de l'échiquier politique

\footnotetext{
6 Yves Lacoste, Géographie du sous-développement, Paris, PUF, 1965, 276 p.

7 Manuel de géographie, $6^{\text {ème }}$, Coll. Marc Vincent, Classiques SUDEL, 1963, (p. 162).

8 Il s'agit du point IV du discours du Président Truman au sujet de l'engagement des Etats-Unis en faveur de l'amélioration et de la croissance des régions «sous-développées ». Voir Gilbert Rist, op.cit, 2001, (p. 121).
} 
international d'après-guerre (la guerre froide) et celui de la décolonisation auraient changé notre regard sur le monde et la formule "sous-développé » est bientôt reprise par les sciences sociales. L'expression n'est pas neutre, elle traduit un changement de perspective dont la portée sera considérable puisqu'une nouvelle lecture ordonnée des espaces et des sociétés s'impose progressivement en référence à des indicateurs géopolitiques (Est/Ouest) et humanitaires (Nord/Sud) jusque là inédits. La faim, la pauvreté, la maladie - ces réalités vieilles comme le monde - sont soudainement «découvertes » et mises en exergue pour réécrire la géographie. Certains annoncent alors la fin des idéologies : «admettre que les populations colonisées étaient dans la misère, c'était, dans une certaine mesure, reconnaître la faillite de cette fameuse mission civilisatrice, alibi idéologique de la colonisation $» 9$. Ainsi, dans un souci d'objectivité et de scientificité retrouvé, un nouveau discours prend forme, chiffres à l'appui : les indicateurs de développement. René Dumont peut dès lors annoncer le mauvais départ de l'Afrique en 196310 et Yves Lacoste rédige une Géographie $d u$ sous-développement deux ans plus tard. La rupture se veut radicale; d'une géographique employée à justifier l'entreprise civilisatrice des anciennes métropoles on en vient à dénoncer le caractère prédateur de la colonisation. Débarrassé de tout préjugé suspect, le concept de développement fait alors son entrée dans les ouvrages scolaires au début des années 1970. Ainsi peut-on lire dans le chapitre d'un manuel de $6^{\text {ème }}$ daté de 1971 , dont l'intitulé est déjà un résumé en soi de ce nouveau regard porté sur le monde

\footnotetext{
9 Yves Lacoste, op.cit, (p.12).

10 René Dumont, L'Afrique noire est mal partie, PUF, Paris, 1963.
}

L'éveil de l'Afrique: "Grâce à l'aide financière des États industrialisés et à la présence des techniciens, des professeurs, des médecins européens, la plupart de ces jeunes États indépendants sont en voie de développement $\gg 11$.

\section{Le développement a-t-il un sens ?}

Alors que les manuels de géographie commencent à diffuser cette nouvelle lecture $\mathrm{du}$ monde, les anthropologues et les sociologues condamnent ce discours qui leur semble relever d'une nouvelle idéologie moderniste aux présupposés suspects, et finissent par déclarer mort-né le concept de développement dès les années 1960. : «Le discrédit dans lequel est tombée [la théorie de la modernisation] vers la fin des années 1960 provient sans doute en partie de la fragilité des résultats obtenus, mais surtout du fait qu'on y a décelé à ce moment un point de vue sociocentrique; les sociétés modernes étant implicitement assimilées aux sociétés occidentales, lesquelles prenaient alors valeur de modèle. Au moment où la décolonisation battait son plein, où le tiersmonde élevait la voix, la théorie de la modernisation paraissait donc comporter des préjugés inacceptables. En outre, elle était perçue comme impliquant un évolutionnisme à la fois peu crédible et peu souhaitable $\gg 12$. La thèse développementaliste est alors rapidement décriée pour avoir substitué à l'idéologie coloniale et sa mission " civilisatrice » une nouvelle idéologie universaliste et moderniste qui présente le danger d'un autre « lissage » du monde et de ses humanités. Et Serge Latouche d'y voir « la poursuite de la

\footnotetext{
11 Extrait du manuel de $6^{\text {ème }}$, La terre. Le continent africain, collection Pernet, Géographie, Hachette, 1971, (p.69).

12 A. Guichaoua \& Y. Goussault, Sciences sociales et développement, Armand Colin, 1993, (p.37).
} 
colonisation par d'autres moyens »13. Le monde est pensé en référence à un même dualisme bâti sur le couple anciennes métropoles/anciennes colonies, parfois dissimulé derrière les apparats d'un nouveau vocabulaire qui se veut plus neutre (Nord, Sud). Mais l'Occident demeure implicitement au centre d'une nouvelle grille de lecture établie en fonction d'une même échelle de valeurs : les sociétés et les continents ne sont plus rangés à l'aune du modèle culturel occidental (primitifs/civilisés) mais à celui de son modèle économique (sousindustrialisés/industrialisés). A l'ancien monde colonial se substitue ici un nouveau monde, pensé et porté par une bonne conscience humanitaire emplie, certes, de bonnes intentions mais terriblement réductrice. Le sous-développement était d'abord synonyme de sous-industrialisation, et fort des théories évolutionnistes fondées sur une représentation linéaire de l'histoire des peuples, on commença par qualifier ces sociétés de « pré-industrielles ». La nouvelle mission dont s'étaient auto investies les anciennes métropoles était dès lors d'aider au passage inévitable et salvateur du Sud à l'âge «supérieur», celui de la modernité et de la société de consommation. En somme, la thèse $\mathrm{du}$ sous-développement relevait d'une fiction : projeter à l'ensemble de l'humanité l'histoire de l'Occident et de sa singulière épopée industrielle commencée au $\mathrm{XV}^{\text {ème }}$ siècle entre Venise et Anvers.

Ainsi, on aurait cru dans les années 1960 à la révélation d'un monde nouveau alors qu'il n'y eut que relecture du même paradigme qui pose toujours l'Occident au centre de l'ordre des choses. Le concept de développement devient alors condamnable pour n'être qu'une nouvelle ruse

13 Serge Latouche, «En finir, une fois pour toutes, avec le développement », in Le Monde diplomatique, mai 2001, (pp.6-7). inconsciente - non un complot sciemment organisé - de la pensée occidentale pour projeter sur les autres (relégués pêle-mêle dans une "périphérie ») le seul éclat de sa civilisation. Le scepticisme de Bernard Bret à ce sujet n'y change rien. Lorsque ce dernier écrit que « le développement ne peut être réduit à l'occidentalisation de la planète dans la mesure où celle-ci a produit à la fois le développement et le sous-développement [...] que l'occidentalisation du monde n'a pas produit le développement, mais l'opposition entre le monde riche et le monde pauvre » il tombe précisément dans le piège du développementalisme dénoncé par Gilbert Rist en s'enfermant dans un schéma de pensée binaire et à ce point banalisé qu'il ne peut concevoir et percevoir le monde autrement qu'en référence à deux situations extrêmes mises bout à bout (développés/sous-développés, riches/pauvres) ${ }^{14}$.

\section{Un évolutionnisme révolu}

L'Afrique serait en retard car elle aurait manqué son départ ${ }^{15}$, 1'Amérique latine produirait de gros efforts et l'Asie afficherait des avancées spectaculaires. Certains sont en bas de l'échelle, d'autres se hissent vers le haut, en tout cas une chose est sûre: tous n'ont pas atteint le même niveau. Quelle est cette course et dans quel sens faudrait-il courir ? Comment prétendre rendre le monde intelligible en recourant à de telles images ? La géographie peut-elle être crédible, et pour combien de temps encore, en donnant à voir de telles représentations où tous les peuples sont mis en compétition et classés au palmarès international d'un jeu du progrès un peu puéril ?

14 Bernard Bret, Le Tiers Monde. Croissance, développement, inégalités, coll. Histege, Ellipses, Paris, 2002, (p. 207), 222p.

15 Référence à René Dumont, op.cit, 1963. 
On retrouve en fait ici un raisonnement empreint de la théorie des étapes de la croissance de Rostow ${ }^{16}$ qui supposait vers la fin des années 1950 l'existence d'un rapport de continuité entre les différents stades économiques. De même, l'opposition sous-développés/développés suggère que toute évolution est condamnée à suivre une seule et même direction pour atteindre un objectif (supposé) commun. Voilà un raisonnement désormais peu convaincant fondé sur l'idée que l'économie de marché serait autorégulée et capable de soumettre à sa logique l'ensemble des secteurs de la société, voire de remodeler à son image les valeurs centrales de cette dernière 17 . Le concept de développement entretient l'illusion d'un monde sans histoire et débarrassé de ses aspérités socioculturelles, un monde engagé dans une seule et même course à sens unique (celui menant à une seule forme de progrès). C'est le mythe de l'occidentalisation achevée et réussie où tous les peuples colonisés, forgés à l'image de quelques sociétés dominatrices auto-proclamées " exemplaires » (converties au monothéisme, à l'économie de marché, à la raison moderne, à l'individualisme), poursuivraient les mêmes dessins. Or, André Guichaoua et Yves Goussault nous rappellent que «les théories de la modernisation et du développement, l'évolutionnisme et le diffusionnisme, ont en commun d'oublier les processus d' « internalisation », c'est-à-dire de décomposition/reconstitution des logiques traditionnelles dans les processus d'innovation » 18 . Et Bertrand Badie d'ajouter que «ces visions développementalistes consomment leurs dernières illusions, tandis que se dissipent

\footnotetext{
16 Gilbert Rist, op.cit.

17 Alain Touraine, Critique de la modernité, Fayard, 1992, 510 p.

18 A. Guichaoua \& Y. Goussault, op.cit, (p.36).
}

les rêves de convergences et le mythe d'un progrès uniforme et continu. Les sciences sociales sont désormais solidement installées dans un post-développementalisme qui présente au moins le mérite d'être plus lucide $\gg 19$. En effet, on sait désormais combien les sociétés africaines, asiatiques et océaniennes, pour être réceptives aux apports extérieurs, n'en sont pas pour autant facilement malléables.

Ni l'Afrique, ni l'Asie, ni le Maghreb... ne sont en retard sur notre voie de développement, pas plus que nous ne sommes en avance sur les leurs. Ce serait oublier l'autonomie des hommes à construire leur destin ou l'irréductibilité culturelle de ces peuples, porteuse d'autres logiques, d'autres valeurs, d'autres rationalités... d'autres projets de société que Louis Dumont appelle holistes ${ }^{20}$. Leur quête du bonheur ne s'est pas arrêtée le jour des indépendances et sans doute prospectent-ils depuis d'autres voies, ni bonnes ni mauvaises, mais si singulières qu'elles devraient plutôt intriguer et susciter un questionnement sur la nature et les raisons de ces «déviances». Mais il faut pour cela adopter un point de vu "décentré ", comme l'appelait de ses vœux Claude Lévi-Strauss, et reconnaître que la recherche d'un mieux vivre est une obsession universelle inscrite dans l'histoire des peuples et accorder à tous un droit à la différence dans cette quête.

Il ne saurait être question ici de tomber dans le piège du culturalisme ou, à l'inverse, de porter un jugement de valeur sur ces "déviances" en alimentant le débat manichéen qui oppose tradition et modernité, trop souvent mis en avant pour

\footnotetext{
19 Bertrand Badie, L'Etat importé, L'occidentalisation de l'ordre politique, Fayard, 1992, 344 p., (p.9).

20 Louis Dumont, Essais sur l'individualisme. Une perspective anthropologique sur l'idéologie moderne, Seuil, 1983, 310 p., (p.37).
} 
tromper l'opinion. L'histoire du monde n'est pas linéaire, sauf à tomber dans les travers d'un discours téléologique qui prétendrait que le « retour en arrière est impossible ... nulle société ne peut aujourd'hui refuser le développement en prétendant rester ellemême pour éliminer misère et famine $»^{21}$. Heureusement, les observations de terrain invitent aujourd'hui à rompre définitivement avec ces images des pays du Sud que l'on dit encore, et sans discernement, empêtrés dans des traditions immuables pour ne pas y avoir relevé les évolutions attendues.

\section{La nouvelle bonne mesure du monde : I'IDH?}

Les critiques précédentes ont fini par être entendues dans les années 1980 et le concept de développement a progressivement muté pour recouvrir aujourd'hui un sens plus large. Aux indicateurs économiques ont été associés de nouveaux critères (inégalité, exclusion et insécurité). Il convient désormais de distinguer la croissance synonyme d'enrichissement économique $d u$ développement entendu comme une nouvelle appréciation qualitative portant sur une amélioration générale des conditions de $\mathrm{vie}^{22}$. Le Programme des Nations Unies pour le Développement (PNUD) a remplacé l'ancienne unité de mesure du développement (le seul PNB par habitant) par l'Indice de Développement Humain voulu plus juste (qui combine l'estimation du pouvoir d'achat, du niveau d'instruction et de l'espérance de vie). Le concept de développement est ainsi redéfini pour désigner «un processus qui permet à des populations entières de passer d'un état de précarité extrême, une insécurité qui touche tous les aspects de leur vie quotidienne

21 Sylvie Brunel, op.cit, (p. 47).

22 Sylvie Brunel, op.cit., (pp.26-32). (alimentaire, politique, sanitaire...) à des sociétés de sécurité, où les hommes ne se demandent pas chaque jour ce qu'ils vont manger le lendemain, peuvent surmonter les caprices de la nature et maîtriser cette dernière, vaincre la maladie, vivre dans des conditions décentes, avoir la possibilité d'exprimer leurs opinions et d'entreprendre librement pour améliorer leur propre sort et celui de leur famille $»^{23}$.

Mais ce repositionnement conceptuel pose à nouveau problème. Il présente, on le craint, les dangers d'une certaine confusion. A une lecture quantitative trop rigide du développement succède une multitude de critères qualitatifs posés là sans qu'aucune grille d'évaluation objective et universelle ne soit et ne puisse être proposée. Voilà autant d'imprécisions qui peuvent laisser libre cours à nombre d'appréciations faites un peu à l'emporte-pièce et où les jugements de valeur seraient vite légion. En effet, la précarité extrême, des conditions de vie décentes, la liberté de parole et d'action peuvent recouvrir des réalités fort différentes chez un montagnard muòng du Nord Vietnam, un pasteur maasaï ou un fermier texan. Quant à «maîtriser la nature », l'aborigène wallaby ne peut s'y résoudre tant son monde est une création parfaite, une œuvre divine à préserver telle qu'elle est et telle qu'elle doit être, où toutes les choses vivantes ont été faites en secret sous la croûte terrestre, comme tout le matériel de l'homme blanc - ses avions, ses fusils, ses Toyota - et toutes les inventions à venir qui dorment sous la surface du sol 24 .

Vouloir brosser le portrait de ce mondelà en termes de sécurité et d'insécurité est également très délicat puisque toute logique de sécurisation s'inscrit dans un ordre rationnel global qu'il faut, là encore, prendre

\footnotetext{
23 Ibid., (p.27-.31).

24 Bruce Chatwin, Le chant des pistes, Grasset, 1988, 411 p., (p.30).
} 
le temps d'explorer au cas par cas pour en saisir tous les ressorts. Frédéric Landy nous rappelle que «la question de la rationalité des paysans du Tiers-Monde souffre [...] d'un vice de forme dès le départ, car elle est mal posée. Il n'y a pas d'ethnie, de société, de groupes sociaux plus rationnels que d'autres: chaque société a $s a$ rationalité (d'où un certain 'déterminisme de civilisation') » 25 . Ainsi, comment apprécier la sécurité alimentaire et sanitaire des Indiens kayapò lorsque celle-ci est assurée par des plantations cachées, dressées au cœur de la forêt amazonienne sur une aire comparable à celle de l'Europe de l'Ouest pour donner lieu à un paysage utile totalement invisible à l'œil inexpérimenté 26 ? De même, la recherche en anthropologie juridique nous enseigne depuis les années 1980 que la sécurité foncière des agriculteurs africains préalable à l'investissement agricole semble être en bien des endroits mieux assurée par des régimes de droit foncier devenus hybrides (ni traditionnels ni modernes) plutôt que par la détention de titres de propriété dûment enregistrés au cadastre sur le modèle occidental 27 . Quant à la sécurité politique, une lecture attentive des ouvrages qui font aujourd'hui référence, tels ceux de Jean-François Bayart, Bertrand Badie, Sophia Mappa et du GEMDEV invitent à émettre des réserves sur les conséquences d'une démocratie politique à

\footnotetext{
25 Frédéric Landy, Paysans de l'Inde du sud. Le choix et la contrainte, Karthala-Institut français de Pondichéry, Paris, 1994, 491 p., (p.458).

26 Emmanuel Lezy, Guyane, Guyanes. Une géographie "sauvage » de l'Orénoque à l'Amazone, Belin, 2000 p.347, (p.59).

27 Voir à ce sujet les travaux réalisés en anthropologie juridique par Etienne Le Roy et ali, L'appropriation de la terre en Afrique, Karthala, Paris, 1991, 359 p. ainsi que La sécurisation foncière en Afrique, Karthala, Paris, 1996, 388 p.
}

l'occidentale pensée et importée sur un simple mode mimétique en terre africaine ${ }^{28}$.

Il n'est pas question ici de nier les difficultés alimentaires, politiques et sanitaires qui existent ici ou là, mais d'éviter les raccourcis en projetant sur l'Autre, et à sa place, des recettes miracles et des formules toutes faites. Parce que l'économie, comme les autres actes accomplis en société (politique, vie familiale, religion) sont une œuvre historique et culturelle globale, le développement humain n'est pas quantifiable. D'ailleurs, Pierre Gourou ne disait-il pas qu'on ne peut traiter du développement dans l'abstrait: "chaque situation locale est particulière et ne peut guère être traitée par des procédures générales...[et] des divagations chiffrées [...] aussi dérisoires que bien d'autres » 29 ?

Ces quelques remarques invitent simplement à nous méfier de nos propres catégories de pensées devant la diversité du monde et du genre humain qui l'habite et le façonne. Car comme le soulignait G. Nicolas O., n'oublions pas que « le véritable sujet de la géographie n'est pas le géographe mais la société qui s'exprime à travers lui ».30 Attention alors à cet eurocentrisme à ce point normalisé qu'il devient invisible depuis l'Occident, mais auquel répondent depuis les années 1980 certains universitaires africains excédés, emmenés

28 Jean-François Bayart, (dir.), La greffe de l'État, Paris, Karthala, 1996, 404 p., Bertrand Badie, L'État importé. L'occidentalisation de l'ordre politique, Paris, Fayard, 1992, 334 p., Sophia Mappa (dir.), Puissance et impuissance de l'État. Les pouvoirs en question au Nord et au Sud, Paris, Karthala, 1996, 466 p., GEMDEV, Les avatars de l'État en Afrique, Paris, Karthala, 1997, 338p.

29 Pierre Gourou, Terres de bonne espérance. Le monde tropical, Terre humaine, Plon, Chapitre 25, 1982, $455 \mathrm{p}$.

30 G.O. Nicolas, L'espace originel, Collection Eratosthène, 1984, $313 \mathrm{p}$. 
par l'historien sénégalais Cheikh Anta Diop, par un afrocentrisme tout aussi radical et inquiétant qui gagne l'Afrique, l'Europe, les Antilles et l'Amérique du Nord 31 .

Afrocentrisme contre eurocentrisme : la vérité en noir et blanc

Avec Antériorité des civilisations nègres (1967), Diop affirmait la primauté du fait nègre dans le processus d'hominisation, l'histoire du peuplement et l'histoire de la civilisation. Dans L'unité culturelle de l'Afrique noire (1959), il traçait les contours culturels respectifs du monde blanc et du monde noir, en posant que le système du matriarcat est fondamentalement « nègre » en dépit d'altérations ou d'apports postérieurs. Avec Nations nègres et culture (1955), il affirmait la négrité de l'Égypte antique et démontrait le complot occidental qui avait masqué cette vérité connue des Anciens. Il y ébauchait une comparaison linguistique que poursuivait Parenté génétique de l'égyptien pharaonique et des langues négro-africaines (1977). L'Afrique noire précoloniale (1960) voulait faire la liaison entre l'Égypte antique et les royaumes africains ruinés par la colonisation. Quant à l'avenir du continent, il se lit dans Les fondements économiques et culturels d'un État fédéral d'Afrique noire (1960). Son dernier ouvrage, enfin, Civilisation ou Barbarie (1981), offre une synthèse de sa pensée et répond à quelques critiques.

Extrait de François-Xavier Fauvelle-Aymar, « Cheikh Anta Diop, ou l'africaniste malgré lui. Retour sur son influence dans les études africaines », in F.-X. Fauvelle-Aymar \& alii, Afrocentrismes. L'histoire des Africains entre Égypte et Amérique, Karthala, Paris, (pp. 27-46), 2000, 402 p.

31 C.A. Diop, Nations nègres et culture. De l'antiquité nègre égyptienne aux problèmes culturels de l'Afrique noire aujourd'hui, Paris, Présence africaine ( $1^{\text {ère }}$ édition 1955). Pour une bibliographie fournie on peut se reporter à l'ouvrage critique de la pensée de Cheikh Anta Diop : F.-X. Fauvelle-Aymar, J.-P. Chrétien, C.-H. Perrot, Afrocentrismes. L'histoire des Africains entre Égypte et Amérique, Karthala, Paris, 2000, 402 p.

\section{L'IDÉOLOGIE GÉOGRAPHIQUE OCCIDENTALE}

La remise en question de la thèse développementaliste dépasse largement le cadre d'une réflexion qui se limiterait à discuter de la validité d'un concept isolé de la géographie. C'est une interrogation globale sur les fondements théoriques et épistémologiques de la discipline qu'il convient désormais de poser pour participer, comme nous y invite Paul Claval, à la reconstruction en profondeur d'un savoir géographique plus rigoureux 32 .

\section{Les dérives de la Nouvelle Géographie}

La thèse développementaliste répondait aux aspirations d'une Nouvelle géographie qui, aux lendemains de la Seconde Guerre mondiale, souhaitait proposer, enfin, une lecture globale du monde et de ses humanités. L'heure était alors à la recherche de lois universelles d'organisation de l'espace pour «ranger le monde». C'est d'abord vers les sciences économiques que l'on se tourna pour opérer des distinctions et proposer des classifications chiffrées.

Cependant, Paul Claval relève combien le portrait du monde ainsi dressé est devenu morne et peu crédible 33 . Réduit à de simples considérations de coûts (coûts des approvisionnements, des expéditions, des informations à centraliser ou à diffuser), le monde serait régi par des logiques de localisation elles-mêmes commandées par d'implacables contraintes économiques. Il faut sans doute déceler dans cette lecture le résultat d'une abstraction théorique longtemps recherchée par une discipline en

32 Paul Claval, «Champs et perspectives de la géographie culturelle dix ans après », in Géographie et cultures, $\mathrm{n}^{\circ} 40$, hiver 2001, (pp.5-28).

33 ibid., (p.25). 
quête de lois universelles pour asseoir son statut scientifique à un moment où celui-ci semblait manquer d'assurance. Mais la théorie aurait conduit à une quantification souvent outrancière appliquée à un monde habité par homo ceconomicus. Ainsi, le concept de développement apparaissait comme le produit d'une lecture matérialiste du monde d'autant plus opportune qu'elle était frappée du sceau séduisant de la nouveauté étayée, qui plus est, d'un arsenal économétrique qui venait à point nommé pour faire montre d'une rationalité instrumentale jugée plus sérieuse.

Mais qu'est-ce que l'économie sinon la transformation de la nature, l'ensemble des actes mis en œuvre pour extraire de celle-ci des ressources, les transformer en biens et richesses afin de les redistribuer, les échanger et les consommer ? ${ }^{34}$. L'anthropologie nous invite, notamment depuis les travaux de Karl Polanyi, George Dalton, Claude Lévi-Strauss et de Maurice Godelier à relever que « dans de nombreuses sociétés l'économie ne présente pas l'aspect de système (séparés) et qu'elle n'opère pas selon une logique propre, indépendante des autres registres $\mathrm{du}$ social $»$. Il faut "relativiser la donne économique qui se pose de plus en plus comme un en-soi apodictique et dont la violence, réelle ou idéelle, tend à nous submerger. La posture anthropologique doit nous permettre d'éviter la naturalisation de l'économique. Nulle immanence dans ce que les hommes élaborent en société, mais bien plutôt le fait de choix culturels »35. L'idéologie moderniste est ici condamnée pour avoir défendu et entretenu une vision erronée des logiques qui fondent la croissance économique, en passant notamment sous silence le rôle déterminant des facteurs

34 Francis Dupuy, Anthropologie économique, Armand Colin, 2001, 192 p., (p.11).

35 ibid., p.5. sociaux et culturels. Dès lors, la théorie évolutionniste de Rostow ne tient plus et vouloir maintenir le discours développementaliste qui s'en inspirait devient suspect.

Disqualifier la pertinence du concept de développement n'est pas une «dérive [...] vers un racisme 'différentialiste', qui dénierait à certains hommes enserrés dans des cultures aliénantes le droit de bénéficier des portées émancipatrices de la modernité $» 36$. Quand finirons-nous par reconnaître que toute culture est aliénante et que cette modernité-là n'a peut-être pas le monopole de l'émancipation des peuples? En somme, ici s'opposent les représentations de deux mondes fantasmatiques qu'il convient de rejeter ; l'un, celui des « traditionalistes romantiques » nostalgiques des sociétés supposées être restées ellesmêmes depuis la nuit des temps mais soudainement - en proie aux effets déstructurant de la société de consommation et de la mondialisation. L'autre, celui des " développementalistes eurocentristes » qui nourrissent le fantasme d'un monde tout entier acquis aux logiques de marché, à une conception univoque et étriquée du progrès.

Que la géographie semble pauvre dans tout ça! Cette science humaine qui oublie les hommes, cette science sociale qui ne dit rien des sociétés où se donnent pourtant à voir toute l'ardeur et l'ingéniosité que mettent les hommes à façonner des villes, des champs, des aéroports... bref, à penser et à produire de l'espace et des territoires. A poursuivre dans cette voie on se condamnerait à la caricature dans l'analyse des rapports qui s'établissent entre l'homme et la nature, et le discours développementaliste en serait devenu l'une des plus manifestes illustrations. Handicap

36 Jean Jacob, «La dérive du tiers-mondisme. Défense de l'identité ou antimodernisme? », in Espace Temps, Sortir du Tiers-monde, $\mathrm{n}^{\circ} 45-46$, 1991, (pp.73-80). 
d'autant plus regrettable qu'il promeut, on peut le craindre, des représentations contraires aux ambitions d'une discipline qui veut expliquer la diversité des hommes et des espaces et montrer comment l'intelligence de 1'Autre, aussi, s'exerce sur la nature.

Il ne s'agit pas non plus de sombrer dans un « rejet fanatique ${ }^{37} \mathrm{du}$ modèle occidental (comme d'aucun autre modèle). L'histoire des peuples n'est pas, et n'a sans doute jamais été, faite de cloisonnements entre civilisations mais bien au contraire d'échanges et d'ouvertures aux influences extérieures. Ce n'est donc pas en termes d'opposition et d'affrontement entre tradition et modernité qu'une lecture lucide et réaliste du monde et des sociétés pourra être menée, mais en termes de combinaisons et de syncrétismes innovants et toujours singuliers au regard desquels le concept de développement devient une nouvelle fois caduc. Le monothéisme se marie très bien à l'animisme sous les tropiques, l'économie de marché n'a pas débouché sur la privatisation des moyens de production dans les campagnes africaines et asiatiques et l'individualisme progresse en même temps que l'ethnicisation des rapports sociaux dans la région des Grands Lacs.

Ainsi, selon Bertrand Badie, l'universalisme naïf ne doit pas pour autant céder la place à un culturalisme dangereux qui figerait les cultures, « forçant leurs traits jusqu'à les installer dans des ghettos, en poussant le relativisme jusqu'à l'absurde... [ce qui condamnerait] tout un chacun à choisir entre la domination et le désordre, entre l'uniformité du modèle occidental et l'éclatement infini des cultures. Une analyse culturelle 'revisitée' et émancipée de telles simplifications peut permettre de penser les rapports interculturels en d'autres termes. Ceux-ci peuvent en effet avoir une

\footnotetext{
37 ibid, 1991.
}

signification toute différente dès lors qu'ils ne sont plus dominés par la dialectique de l'importation et de la résistance, de la négation de la différence et de l'exclusion [...] Cette multiculturalité n'a pas aboli les cultures : au contraire, elle a appelé à une dédramatisation de la différence. L'enjeu qui se profile, à partir de cette observation, tient à l'institutionnalisation de cette pluralité, à l'échelle des rapports mondiaux, comme à celle des rapports nationaux $» 38$.

«La cacophonie d'un monde qui ne parvient ni à unifier ses règles du jeu ni à faire leur place aux différences constitue sans nul doute la plus lourde des menaces qui pèsent sur l'humanité »39.

\section{Géographie culturelle ou culture géographique?}

Faut-il accepter qu'une telle analyse relève de cette branche spécifique, voire exotique, de la géographie que l'on dit «culturelle»? Suffit-il en effet de relever l'intrication de la culture, de la politique et de l'économie dans la production de l'espace pour verser immédiatement dans l'excentricité ?

Deux raisons majeures s'opposent à qualifier cette approche d'excentrique, sauf à ne retenir que l'acception mécaniste du Petit Robert: «L'excentrique permet de transformer un mouvement rotatif en un mouvement de va et vient et inversement $» 40$. Voilà en effet une image assez satisfaisante du détour anthropologique souhaité par Georges Balandier, susceptible de nous procurer, par

\footnotetext{
38 Bertrand Badie, op.cit, 1992, (pp.315-316).

39 ibid., $4^{\text {ème }}$ page de couverture.

${ }^{40}$ Le Petit Robert, tome 1, 1991, p. 723.
} 
l'Autre interposé, un regard distancié41. Cette réserve mise à part, l'excentricité au sens commun de marginalité n'est pas recevable.

Tout d'abord, parce que la culture est immanente à toute analyse, on ne peut penser le monde sans recourir à ses propres valeurs, marquées du sceau de notre civilisation et posées comme un filtre interprétatif qui donne sens aux choses. Comme le disait Gilles Sauter «supposons par hypothèse le géographe disculpé, dans sa fonction d'intellectuel et reconnu porteur d'un regard socialement transparent. Mais alors sa force propre de voir et de sentir, d'appréhender et de coder, puis de traduire...ne dresse t-elle pas elle-même une barrière ?»42. La neutralité ou l'objectivité dans la mise en ordre du monde serait alors impensable et impossible car cette dernière déboucherait sur un agencement inintelligible et inexpressif. Ainsi, aucun discours géographique ne peut déroger à la règle culturelle, y compris, bien sûr, la géographie économique et la thèse développementaliste qui en émane, enchâssées elles-mêmes dans une idéologie moderniste eurocentrique née de la philosophie mécaniste des Lumières. A ce titre donc, la « géographie culturelle » n'est pas plus culturelle que les autres branches de la discipline. Sans doute vaudrait-il mieux alors inverser l'ordre des mots et parler de « culture géographique » pour rappeler que la culture est tout autant objet que sujet d'étude en sciences sociales. A ce titre donc, dire que la culture est ce qui reste d'inexpliqué quand on a tout expliqué (Joël Bonnemaison) $)^{43}$ est discutable. La culture

\footnotetext{
${ }^{41}$ Francis Dupuy, op.cit., 2001, (p.5).

42 Gilles Sautter, "Le paysage comme connivence », Hérodote $\mathrm{n}^{\circ} 16,1979$.

43 Joël Bonnemaison, Géographie culturelle, CTHS, 2000, 152 p.
}

n'est-elle pas, au contraire, au commencement de l'explication ?

$\mathrm{La}$ deuxième réserve s'inspire des recherches en anthropologie économique. S'il est admis aujourd'hui que la culture en général, et les relations sociales en particulier, englobent la manière dont l'homme exerce son emprise sur la nature (prélèvement, transformation, production, distribution, consommation de la ressource), les rapports économiques (compétition et concurrence) ne s'inscrivent pas alors dans la nature des choses mais bien dans un ordre social. Chez les uns, la loi dominante est celle du «marché », chez les autres celle de la « réciprocité », ailleurs encore celle de la « redistribution $» 44$. Encore que la loi du marché elle-même peut parfois souffrir quelques entorses; la morale, les pratiques religieuses, la mode, le respect de l'environnement invitent chez les Occidentaux à l'observation de certains comportements qui outrepassent une rationalité comprise comme devant aller vers la seule maximisation du profit. C'est finalement le respect des règles d'un «jeu de société » toujours savamment orchestré qui compte, communément résumé par ce que nous appelons un « savoir-vivre », gage essentiel d'intégration et de respectabilité.

De même, le producteur de coton indien et le planteur de café ougandais doivent composer constamment avec les éléments sociaux érigés par leurs cultures. Le respect des interdits totémiques, l'importance des dépenses festives et funéraires, l'origine de la parenté, le nombre d'épouses et de descendants... procurent un statut à défendre ou à acquérir, parfois de manière dispendieuse pour observer les règles de bienséances auxquelles nul ne peut échapper en société. Voilà un autre univers contraignant et à la fois valorisant, en

\footnotetext{
44 Karl Polanyi, Trade and Market in the early Empires, 1957.
} 
fonction duquel des stratégies économiques ont émergé, des choix de production ont été privilégiés...et des politiques de "développement» ont échoué. La maximisation du profit ne sera pas ici forcément socialement rationnelle ; elle peut bien au contraire s'apparenter à un suicide. Marshall Sahlins résume admirablement ceci en écrivant: " la pauvreté ne constitue pas en une faible quantité de biens, ni simplement en une relation entre moyens et fins ; c'est avant tout une relation d'homme à homme, un statut social » 45 . Paul Claval ne disait-il pas en effet qu'il faut mettre l'accent sur les facteurs psychologiques et culturels (engendrés par les systèmes de valeurs collectives ou produits de façon plus aléatoire par les individus) intervenant dans l'explication des organismes géographiques : «Il faut se familiariser avec le sens (qu'ils) donnent aux rôles, aux lieux, aux choses. Il faut pénétrer l'intérieur des systèmes de valeurs »46. Et Guy Di Méo d'ajouter "C'est donc, me semble-t-il, vers une imbrication des faits de société et des valeurs culturelles, gérés par des lois sociales qu'il faut nous orienter. L'espace subit de plein fouet les effets déterminants de ce complexe causal que véhiculent au quotidien les représentations et les pratiques sociales. Il contribue aussi, en retour, à le forger $\gg 47$.

Ainsi, replacer le fait culturel au centre de l'explication des phénomènes géographiques ne vise pas à donner une coloration plus sympathique et chaleureuse à l'analyse. C'est une réflexion épistémologique en profondeur qu'il

\footnotetext{
45 Citation extraite de Francis Dupuy, op.cit., 2001, (p.31).

46 Guy Di Méo, «L'explication sociale en géographie », in Explications en géographie. Démarches, stratégies et modèles, SEDES, 2001, (p.110).

47 ibid., (p 111).
}

convient désormais d'engager. Pourquoi faudrait-il en effet se convaincre que les dynamiques agraires, l'urbanisation, les bouleversements sociaux et politiques contemporains répondent partout aux mêmes causes et visent les mêmes objectifs suivant les mêmes logiques ? On peut lire, par exemple, que l'urbanisation gagne l'Afrique, mais n'est-ce pas plutôt l'Afrique qui prend la ville déposée là par l'Occident et l'investit avec frénésie pour la remodeler à son image, bouleversant à sa manière tous les schémas établis, malmenant toutes les définitions d'usage de l'urbain et du rural, de la ville et du citadin ? L'urbanisation mise en germe par le colonisateur sur plusieurs continents a transformé la géographie de bien des pays autant qu'elle a été transformée en retour par leur géographie. A Brazzaville, Roland Pourtier constate que la ville est déconstruite pour devenir un théâtre où se donne à voir en résumé une géographie régionale et nationale 48 . La signification de la ville semble avoir muté pour devenir un espace disputé selon des modalités inédites du fait de la convergence de multiples fronts de peuplement. Brazzaville apparaît comme le prolongement incongru de mondes ruraux attenants où la promiscuité avive toutes les tensions pour la maîtrise des ressources et des services (terrain, eau, emploi, information, santé...). En somme, le fait culturel semble bien avoir remodelé le fait urbain. De même, Jean-Luc Piermay souligne dans un article intitulé «L'invention de la ville en Afrique sudsaharienne » combien l'urbanité africaine doit être repensée et réévaluée par la géographie pour ne pas tomber dans le piège de la naturalisation de l'espace urbain, prémisse d'un déterminisme qui conduirait à

\footnotetext{
48 Roland Pourtier, « Brazzaville dans la guerre : crise urbaine et violences politiques », in Annales de géographie, $\mathrm{n}^{\circ} 611$.
} 
discourir dans l'abstrait 49 . Sans doute alors faudra t-il bientôt rompre avec cette idée déjà bien ancrée d'un monde où l'urbanisation annoncée sur tous les continents véhicule une image réductrice de ces regroupements bigarrés qui résultent pourtant de l'invention déroutante de citadinités retravaillées de l'intérieur.

«Le concept d'invention porte à
considérer, au-delà des afro-
pessimismes, des sociétés qui se
créent et qui créent leurs espaces
dans les conditions les plus
difficiles et les plus ingrates qui
soient. [...] Il pose enfin la
redoutable question de l'avenir de
la ville africaine, en incitant à faire
confiance en l'inventivité des
sociétés et des individus placés dans
des conditions impossibles, tout en
obligeant à la plus grande prudence
face à des assemblages à venir
imprévisibles et à coup presque sûr
baroques et surprenants 50

\section{Le monde enchanté de nos idéologies}

Le concept de développement est né d'une théorie évolutionniste du progrès synonyme d'abondance, de liberté et de bonheur qui ne semble désormais plus faire recette. Alain Touraine rappelle que cette théorie n'a été qu'une idéologie constamment démentie par 1'histoire $^{51}$ car le destin de l'homme n'est inscrit nulle part, et Jacques Ruffié d'ajouter: "Cela ne doit ni surprendre, ni inquiéter. La force de l'homme n'est pas d'être dépourvue d'un

49 Jean-Luc Piermay, «L'invention de la ville en Afrique sud-saharienne ", in Historiens \& géographes, n³79, juillet-août 2002, pp. 153-159.

50 ibid, p. 158 .

51 Alain Touraine, op.cit. destin inexorable : c'est de le savoir » 52 . Ce dernier allant jusqu'à prédire une crise qui pourrait déboucher sur l'effondrement de nos civilisations, même sur la disparition de notre espèce : « Nous sommes à la croisée des chemins; nous pouvons retourner à l'animalité », sauf à espérer que l'Occident se voit, enfin, tel qu'il est, non tel qu'il croit être 53 .

C'est donc un appel à une géographie décentrée qui est lancé ici, ou le souhait de voir se développer une science qui rendrait compte avec lucidité, recul et sens critique de la manière toujours singulière dont l'homme, ici et là, vit le monde ; c'est-à-dire le pense puis le conceptualise pour enfin s'y projeter et l'investir. Eric Dardel ne disait-il pas que la réalité géographique est celle des mondes vécus de 1'humanité 54 ? En somme, il s'agirait de prendre le monde comme le produit de représentations diverses à partir desquelles les peuples appréhendent, transforment et aménagent une nature biologique. Le projet illusoire d'objectivation du monde, entendu comme une réalité propre - une matérialité extérieure imposée à l'homme - serait alors dépassé par une approche plus rigoureuse d'objectivation des représentations qui font le monde, comme l'idéologie développementaliste, entre autres, l'a fait depuis les années 1950 en Occident.

Voici l'erreur dans laquelle nous a entraîné la Nouvelle géographie en prétendant avoir réussi l'impossible : dégager des lois d'organisation de l'espace qui transcenderaient toutes les idéologies pour, en somme, ramener ce dernier à une simple réalité matérielle objectivable. La proposition est absurde car elle omet

\footnotetext{
52 Jacques Ruffié, De la biologie à la culture, Vol 2, Champs-Flammarion, 1983, 334 p.

53 Ibid., pp.302-303.

54 Eric Dardel, L'homme et la terre, première édition 1952, rééd. CTHS, Paris, 1990.
} 
d'expliciter les fondamentaux culturels dont elle est elle-même pétrie : ceux hérités d'une révolution intellectuelle réalisée au tournant des XVII ${ }^{\text {ème }}$ et XVIII ${ }^{\text {ème }}$ siècles en Occident et aux termes de laquelle ont été instituées, codifiées et standardisées de nouvelles représentations $\mathrm{du}$ réel qui faisaient de l'espace et du temps des substrats neutres et isomorphes, constitués d'un maillage de repères d'une valeur supposée analogue. Ces représentations ont été à ce point normalisées qu'on a depuis oublié qu'elles ne sont qu'une nouvelle vue de l'esprit, des conventions abstraites fondées sur des calculs astronomiques (longitudes, latitudes, le système métrique, le temps horaire). Mais la distance métrique et le temps horaire sont progressivement devenus l'alpha et l'oméga d'une nouvelle " imagerie du réel ». Toutefois, nulle révélation dans tout ça, mais " réidéalisation » ou simple invention d'une nouvelle perception géométrique et chronométrique de l'espace et du temps qui, désormais, structure largement nos représentations. En résumé, c'est par un curieux jeu d'esprit que l'homme des Lumières et ses héritiers se sont persuadés de l'objectivité des règles et des codes qu'ils ont eux-mêmes inventés pour repenser le monde, et Denis Guedj d'y voir un véritable «coup d'État» de la science moderne 55 .

Reprenant les travaux de Paul Bohannan 56 , Etienne Le Roy nous dit que dans certaines sociétés caractérisées par l'animisme et le communautarisme, l'espace prend corps à partir de lieux particuliers, sièges des divers pouvoirs sur les génies, les eaux, les terres, les arbres ou les hommes, les animaux ou les minéraux, selon des

\footnotetext{
55 Denis Guedj, Le mètre du monde, Seuil, 2000, 330 p., (p.82).

56 Paul Bohannan, «Land, Tenure and LandTenure », African Agrarian System, Oxford, Oxford University Press, 1963, (p.106).
}

distinctions fonctionnelles qui permettent à chaque groupe d'exercer, selon son ordre d'arrivée et son activité, des attributs particuliers. Chaque fonction impliquant une maîtrise particulière de l'espace et cette maîtrise étant analogue à une puissance « magnétique » s'exerçant à partir de chacun des lieux, sièges de ces pouvoirs fonctionnels, la représentation de l'espace est «topocentrique $" 57$. Des observations analogues faites chez les populations insulaires de Mélanésie invitent Joël Bonnemaison à conclure également que notre conception de la géographie relève bien d'une idéologie occidentale mise en forme par le pouvoir moderne plus qu'elle n'exprime l'universalité des formes de territorialité ou des représentations de l'espace 58 .

D'ailleurs, l'eurocentrisme dominant dans le discours géographique moderne n'est-il pas également l'expression d'un autre topocentrisme, comme l'atteste notre vocabulaire : Proche-Orient, ExtrêmeOrient, Occident... développé/sousdéveloppé. Voir et penser le monde à l'aune de ses propres valeurs revient en effet, ni plus ni moins, à procéder comme le font les sociétés caractérisées par l'animisme et le communautarisme dont parle Etienne Le Roy. Preuve serait donc faite que la représentation du monde et de l'espace instituée depuis deux siècles en Occident n'a pas mené à l'objectivation du monde et à la rationalisation de nos perceptions. Elle aurait simplement marqué l'avènement d'un bien curieux et paradoxal "topocentrisme euclidien", produit d'une nouvelle instrumentation idéologique de nos représentations.

57 Etienne Le Roy, L'appropriation de la terre en Afrique noire, 1991, Karthala, 359 p., (p.15).

58 Joël Bonnemaison, «L'espace réticulé. Commentaire sur l'idéologie géographique », in Tropiques, lieux et liens, ORSTOM, 1989, (pp.500510). 


\section{Conclusion}

Est-ce à dire alors que le monde du géographe est condamné à n'être qu'un produit discursif, ou la mise en mots et en images de nos idéologies du moment ? En d'autres termes, si la culture gouverne irrémédiablement notre perception du réel, comment prétendre à l'objectivité ? Mais cessons de croire que l'objectivité scientifique se résume à l'impression sensible. Le rôle de la science est de dépasser l'objectivité absolue pour donner sens aux choses ${ }^{59}$ : elle n'a pas à prendre le monde en photo, mais à l'expliquer. Le rôle du scientifique, moins ambitieux mais sans doute plus utile à la collectivité humaine, ne serait plus dès lors d'être un donneur de vérités ni un ambassadeur d'idées, mais un donneur de sens et un passeur d'idées pour aider à une meilleure acceptation de l'Autre dans sa différence et pour sa différence. Ainsi, il me semble que prendre conscience des fondements idéologiques à partir desquels nous structurons nos représentations $\mathrm{du}$ réel serait déjà la première étape d'une démarche mieux assurée et plus rigoureuse dans la mesure où celle-ci permettrait de distancier le sujet de l'objet. Car c'est en prenant préalablement conscience de ses propres représentations et en les interrogeant par la confrontation avec d'autres que le géographe sera à-même de les mettre en perspective pour ensuite nous expliquer en quoi et pourquoi elles diffèrent. Louis Dumont nous invitait déjà à produire cet effort en déclarant que pour dépasser le fondement familier et implicite de notre discours ordinaire, il nous faut mettre en perspective notre culture en la contrastant avec d'autres cultures. C'est seulement ainsi que nous pouvons voir la civilisation occidentale dans son unité et sa

59 Henri Lefebvre, Méthodologie des sciences, Anthropos, Paris, 2002. spécificité60. Ainsi, le "relativisme culturel" ne me semble pas être une dérive dangereuse, il n'invalide pas la recherche scientifique, pas davantage du moins que l'anthropologie économique, politique, juridique ou sociale. Le relativisme culturel en géographie vise bien au contraire à déconstruire les modes de représentation du réel pour mettre à plat les logiques élémentaires - culturelles - qui président à la production de l'espace, tant chez nous que chez l'Autre.

"Il conviendrait non pas seulement d'étudier l'histoire de l'espace, mais celle des représentations, ainsi que celle de leurs liens entre elles, avec la pratique, avec l'idéologie. Une telle histoire comporterait donc la genèse de ces espaces, mais surtout de leurs connexions, distorsions, déplacements interférences et de leurs liens avec la pratique spatiale des sociétés. Que savons-nous en Europe, avec les concepts occidentaux pour instrument, du mode de production asiatique, de son espace, de ses villes, et du rapport entre la ville et la campagne? Connaissons-nous les idéogrammes qui, paraît-il, donnent ce rapport en le figurant?»61.

\footnotetext{
60 Louis Dumont, op. cit.

61 Henri Lefebvre, La production de l'espace, Anthropos, 1974, $4{ }^{\text {ème }}$ éd., 2000.
} 
